\title{
Indium(III) chloride - catalyzed Michael addition of thiols to chalcones : a remarkable solvent effect
}

\author{
Brindaban C. Ranu, ${ }^{*}$ Suvendu S. Dey, and Sampak Samanta \\ Department of Organic Chemistry, Indian Association for the Cultivation of Science, Jadavpur, \\ Calcutta - 700 032, India \\ E-mail: ocbcr@iacs.res.in
}

Dedicated to Dr. A. V. Rama Rao on the occasion of his $70^{\text {th }}$ birthday

(received 28 Aug 04; accepted 23 Sep 04; published on the web 26 Sep 04)

\begin{abstract}
Indium(III) chloride in methanol efficiently catalyzes Michael addition of aromatic and aliphatic thiols to chalcones and related compounds. This reaction is remarkably solvent selective and it does not proceed in conventional solvents such as tetrahydrofuran, methylene chloride and water.
\end{abstract}

Keywords: Michael addition, thiol, chalcone, indium(III) chloride, solvent effect

\section{Introduction}

The Michael addition of thiols to electron deficient alkenes is a very useful process for making carbon-sulfur bond. This addition to chalcone derivatives is very interesting and challenging as it is less facile compared to the addition to aliphatic acyclic enones and thus it is not always satisfactory with the conventional reagents used for general 1,4-addition. Recently, a number of procedures involving a variety of catalysts such as synthetic diphosphate $\mathrm{Na}_{2} \mathrm{CaP}_{2} \mathrm{O}_{7},{ }^{1 \mathrm{a}}$ natural phosphate, ${ }^{1 \mathrm{~b}} \mathrm{InBr}_{3},{ }^{1 \mathrm{c}}$ fluorapatite, ${ }^{1 \mathrm{~d}}$ have been reported.

Indium halides, particularly indium(III) chloride have emerged as a potent Lewis acid for various organic transformations in recent times. ${ }^{2}$ As a part of our interest in indium(III) chloride catalyzed reactions ${ }^{3}$ we discovered that although $\mathrm{InCl}_{3}$ has been used in the Michael addition of silylenol ethers, ${ }^{4 \mathrm{a}}$ amines, ${ }^{4 \mathrm{~b}}$ pyrroles ${ }^{4 \mathrm{c}}$ and indoles ${ }^{4 \mathrm{~d}}$ to electron-deficient alkenes no report has been found demonstrating the use of $\mathrm{InCl}_{3}$ for addition of thiols to chalcones and related systems. This prompted us to investigate this reaction and we observed that very interestingly $\mathrm{InCl}_{3^{-}}$ catalyzed addition of thiol to chalcone derivatives is very much solvent dependent and it does not proceed at all in conventional solvents like $\mathrm{CH}_{2} \mathrm{Cl}_{2}$, THF and $\mathrm{H}_{2} \mathrm{O}$ usually chosen for indium halide mediated reactions. ${ }^{3,4}$ However, we discovered that the reaction goes very efficiently in dry methanol (Scheme 1). 


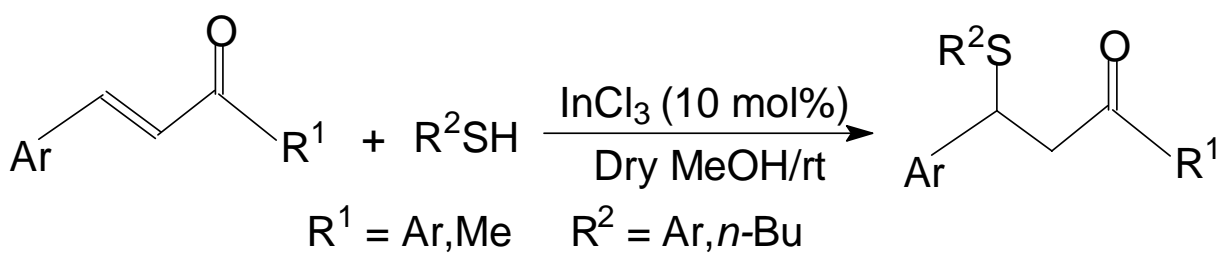

\section{Scheme 1}

\section{Results and Discussion}

The experimental procedure is very simple. A solution of chalcone and thiol in dry methanol was stirred at room temperature in the presence of a catalytic amount of indium(III) chloride. Usual work up with ether furnished the product.

Aliphatic as well as aromatic thiols underwent facile additions to a variety of chalcones by this procedure to provide the corresponding adducts in high yields. The results are summarized in Table 1. The electron withdrawing and electron donating substituents on the aromatic ring of the chalcone derivatives did not influence the progress of reaction. Additionally, additions of thiophenols with different substitutions also proceed efficiently. A vinylogous chalcone (entry 12) underwent bis-additions to provide the corresponding bis-adduct. This procedure also works well with chalcone like systems where the other ends are Me in place of $\mathrm{Ph}$ (entries 13-15).

The reactions are in general, considerably fast, clean and high yielding. The crude products obtained are sufficiently pure and purification by filtration through a short column of silica gel is enough to get pure samples in most of the reactions. All the products were characterized by spectroscopic data (IR, ${ }^{1} \mathrm{H}$ and ${ }^{13} \mathrm{C} \mathrm{NMR}$ ) together with elemental analysis for new compounds. It has been observed that the addition does not proceed at all in the absence of indium(III) chloride. 
Table 1. Michael addition of thiols to chalcone derivatives and related systems

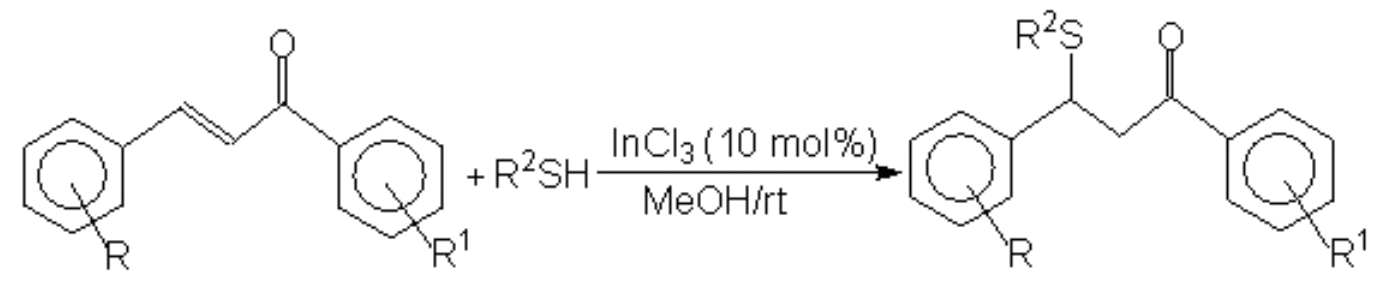

\begin{tabular}{|c|c|c|c|c|c|c|}
\hline Entry & $\mathrm{R}$ & $\mathrm{R}^{1}$ & $\mathrm{R}^{2}$ & Time(h) & Yield(\%) ${ }^{a}$ & Ref \\
\hline 1 & $\mathrm{H}$ & $\mathrm{H}$ & $n$-Bu & 1.0 & 92 & \\
\hline 2 & $\mathrm{H}$ & $\mathrm{H}$ & $\mathrm{Ph}$ & 1.0 & 95 & $1 \mathrm{a}$ \\
\hline 3 & $\mathrm{H}$ & $\mathrm{H}$ & $\left(0-\mathrm{NH}_{2}\right) \mathrm{C}_{6} \mathrm{H}_{4}$ & 2.0 & 72 & $1 \mathrm{a}$ \\
\hline 4 & $\mathrm{H}$ & Me & $\mathrm{Ph}$ & 2.5 & 72 & 6 \\
\hline 5 & $\mathrm{H}$ & Me & $n$-Bu & 3.0 & 61 & \\
\hline 6 & $(p-C l)$ & $\mathrm{H}$ & $n$-Bu & 1.5 & 86 & \\
\hline 7 & $(p-\mathrm{Cl})$ & $\mathrm{H}$ & $(p-C) \mathrm{C}_{6} \mathrm{H}_{4}$ & 2.0 & 90 & \\
\hline 8 & $\left(p-\mathrm{NO}_{2}\right)$ & $\mathrm{H}$ & $n-\mathrm{Bu}$ & 2.5 & 74 & \\
\hline 9 & $\left(p-\mathrm{NO}_{2}\right)$ & $\mathrm{H}$ & $(p-\mathrm{Cl}) \mathrm{C}_{6} \mathrm{H}_{4}$ & 1.5 & 86 & \\
\hline 10 & $(p-\mathrm{OMe})$ & $\mathrm{H}$ & $n$-Bu & 2.5 & 75 & \\
\hline 11 & (p-OMe) & $\mathrm{H}$ & $\mathrm{Ph}$ & 2.5 & 69 & $1 d$ \\
\hline 12 & & & $n$-Bu & 2.0 & $72^{b}$ & 7 \\
\hline 13 & & & $n-\mathrm{Bu}$ & 1.75 & 84 & \\
\hline 14 & & & $\mathrm{Ph}$ & 2.0 & 80 & 8 \\
\hline 15 & $T y$ & & $\left(0-\mathrm{NH}_{2}\right) \mathrm{C}_{6} \mathrm{H}_{4}$ & 2.0 & 72 & \\
\hline
\end{tabular}

${ }^{a}$ The yields refer to those of pure isolated products characterized by spectro-scopic ( IR, $1 \mathrm{H}$ and 13C NMR ) data. ${ }^{\mathrm{b}}$ The yield refers to bis - addition product. 
The reactions are accomplished most efficiently with $10 \mathrm{~mol} \%$ of $\mathrm{InCl}_{3}$ with respect to chalcone; however with the lower amount than that the reactions are found to be slow. The influence of methanol as solvent towards progress of this $\mathrm{InCl}_{3}$ catalyzed reaction, though interesting is not unprecedented. ${ }^{5}$

In conclusion, the present procedure catalyzed by indium(III) chloride provides an efficient methodology for the Michael addition of thiols to chalcone derivatives and related systems. The mild reaction conditions (room temperature), simple operation, high yields of adducts, relatively low catalyst loading and much lower cost of indium(III) chloride compared to indium(III) bromide that has been used earlier for this conjugate addition ${ }^{1 \mathrm{c}}$ make this procedure an attractive alternative to the existing procedures. Moreover, this work demonstrates the potential of indium(III) chloride as a Lewis acid and the remarkable effect of solvent in promoting catalytic activity.

\section{Experimental Section}

General Procedures. NMR spectra were recorded on a Bruker DPX 300 instrument at $300 \mathrm{MHz}$ for ${ }^{1} \mathrm{H}$ and at $75 \mathrm{MHz}$ for ${ }^{13} \mathrm{C}$ NMR in $\mathrm{CDCl}_{3}$ solutions. IR spectra were measured on a FT-8300 Shimadzu spectrometer in neat for liquids and in $\mathrm{KBr}$ pellet for solids. Methanol was distilled over sodium metal. Chalcones were prepared by standard procedure. ${ }^{9}$ Thiols were distilled before use. Indium(III) chloride (98\%) was purchased from Aldrich and was used as such.

\section{Representative experimental procedure for Michael addition (entry 1, Table 1)}

A mixture of chalcone (208 mg, $1 \mathrm{mmol})$, butanethiol $(117 \mathrm{mg}, 1.3 \mathrm{mmol})$ in dry methanol (1.5 $\mathrm{mL}$ ) was stirred at room temperature in the presence of a catalytic amount of indium(III) chloride (10 mol\% with respect to chalcone, $22 \mathrm{mg}$ ) for $1 \mathrm{hr}$. The reaction mixture was then extracted with ether and the ether extract was washed with $\mathrm{H}_{2} \mathrm{O}$, brine and dried $\left(\mathrm{Na}_{2} \mathrm{SO}_{4}\right)$. Evaporation of solvent furnished the crude product that was purified by column chromatography over silica gel (hexane/ether 96:4) to give pure adduct, 1,3-diphenyl-3-(thiobutyl)propan-1-one (274 mg, 92\%) as a colourless oil, IR 3028, 2952, 1675, $1603 \mathrm{~cm}^{-1} ;{ }^{1} \mathrm{H}$ NMR $\delta 0.82(\mathrm{t}, J=7.2 \mathrm{~Hz}, 3 \mathrm{H}), 1.26-$ $1.34(\mathrm{~m}, 2 \mathrm{H}), 1.39-1.56(\mathrm{~m}, 2 \mathrm{H}), 2.27-2.37(\mathrm{~m}, 2 \mathrm{H}), 3.52(\mathrm{~d}, J=7.0 \mathrm{~Hz}, 2 \mathrm{H}), 4.55(\mathrm{t}, J=7.0 \mathrm{~Hz}$, 1H), 7.17-7.54 (m, 8H), 7.88-7.91 (m, 2H); ${ }^{13} \mathrm{C}$ NMR $\delta 14.0,22.4,31.5,31.7,44.7,45.9,127.6$, 128.3 (2C), 128.5 (2C), 128.8 (2C), 128.9, 129.0, 133.6, 137.3, 142.7, 197.4. Anal. calcd for $\mathrm{C}_{19} \mathrm{H}_{22} \mathrm{OS}: \mathrm{C}, 76.47$; H, 7.43. Found: $\mathrm{C}, 76.31 ; \mathrm{H}, 7.28$.

This procedure is followed for all the reactions listed in Table 1. Several products are known compounds and were identified by spectroscopic data (IR, ${ }^{1} \mathrm{H}$ and ${ }^{13} \mathrm{C}$ NMR) which are in good agreement with those reported (references in Table 1). The spectroscopic and analytical data of a few products whose spectroscopic data are not readily available are provided here in order of their entries in Table 1. 
1-(4-Methylphenyl)-3-phenyl-3-thiobutylpropan-1-one (entry 5). White crystal; m.p. $58{ }^{\circ} \mathrm{C}$ (hexane-ether 4:1); IR 3028, 2952, 1674, $1606 \mathrm{~cm}^{-1} ;{ }^{1} \mathrm{H}$ NMR $\delta 0.83(\mathrm{t}, J=7.3 \mathrm{~Hz}, 3 \mathrm{H}), 1.26-$ $1.37(\mathrm{~m}, 2 \mathrm{H}), 1.42-1.50(\mathrm{~m}, 2 \mathrm{H}), 2.27-2.36(\mathrm{~m}, 2 \mathrm{H}), 2.37(\mathrm{~s}, 3 \mathrm{H}), 3.47(\mathrm{~d}, J=7.1 \mathrm{~Hz}, 2 \mathrm{H}), 4.54$ $(\mathrm{t}, J=7.1 \mathrm{~Hz}, 1 \mathrm{H}), 7.20-7.32(\mathrm{~m}, 5 \mathrm{H}), 7.41(\mathrm{~d}, J=8.2 \mathrm{~Hz}, 2 \mathrm{H}), 7.81(\mathrm{~d}, J=8.2 \mathrm{~Hz}, 2 \mathrm{H}) ;{ }^{13} \mathrm{C}$ NMR $\delta$ 14.0, 22.0, 22.3, 31.6, 31.7, 44.8, 45.7, 127.5, 128.2 (2C), 128.6 (2C), 128.9 (2C), 129.7 (2C), 134.8, 142.8, 144.4, 197.0. Anal. calcd for $\mathrm{C}_{20} \mathrm{H}_{24} \mathrm{OS}: \mathrm{C}, 76.88$; H, 7.74. Found: C, 77.09; H, 7.69.

3-(4-Chlorophenyl)-1-phenyl-3-thiobutylpropan-1-one (entry 6). Colorless liquid; IR 2956, 2869, 1687, $1596 \mathrm{~cm}^{-1}$; ${ }^{1} \mathrm{H}$ NMR $\delta 0.84(\mathrm{t}, J=7.2 \mathrm{~Hz}, 3 \mathrm{H}), 1.20-1.57(\mathrm{~m}, 4 \mathrm{H}), 2.26-2.40(\mathrm{~m}$, $2 \mathrm{H}), 3.49(\mathrm{~d}, J=7.0 \mathrm{~Hz}, 2 \mathrm{H}), 4.52(\mathrm{t}, J=7.0 \mathrm{~Hz}, 1 \mathrm{H}), 7.24-7.57(\mathrm{~m}, 7 \mathrm{H}), 7.89$ (d, $J=7.8 \mathrm{~Hz}$, 2H); ${ }^{13} \mathrm{C}$ NMR $\delta 14.0,22.3,31.5,31.6,43.9,45.7,122.9$ (2C), 128.9, 129.0, 129.1 (2C), 129.7 (2C), 133.8, 137.0, 141.3, 143.7, 197.1. Anal. calcd for $\mathrm{C}_{19} \mathrm{H}_{21} \mathrm{OSCl}: \mathrm{C}, 68.55 ; \mathrm{H}, 6.36$. Found: C, 68.38; H, 6.30.

3-(4-Chlorophenyl)-3-(4-chlorothiophenyl)-1-phenylpropan-1-one (entry 7). White crystal; m.p. 84-86 ${ }^{\circ} \mathrm{C}$ (hexane-ether 4:1); IR 3061, 1685, 1473, $688 \mathrm{~cm}^{-1} ;{ }^{1} \mathrm{H}$ NMR $\delta 3.58$ (d, $J=7.1 \mathrm{~Hz}$, $2 \mathrm{H}), 4.88(\mathrm{t}, J=7.1 \mathrm{~Hz}, 1 \mathrm{H}), 7.16-7.58(\mathrm{~m}, 11 \mathrm{H}), 7.88(\mathrm{~d}, J=7.8 \mathrm{~Hz}, 2 \mathrm{H}) ;{ }^{13} \mathrm{C}$ NMR $\delta 44.8$, 48.3, 128.4 (2C), 128.9 (2C), 129.0, 129.1 (2C), 129.4 (2C), 129.5 (2C), 130.8, 133.9, 134.5, 134.8 (2C), 137.5, 140.1, 196.8. Anal. calcd for $\mathrm{C}_{21} \mathrm{H}_{16} \mathrm{OSCl}_{2}$ : C, 65.12; H, 4.16. Found: C, 64.94; H, 4.04.

3-(4-Nitrophenyl)-1-phenyl-3-thiobutylpropan-1-one (entry 8). Colorless oil; IR 2956, 2871, 1687, 1519, $1346 \mathrm{~cm}^{-1}$; ${ }^{1} \mathrm{H}$ NMR $\delta 0.84(\mathrm{t}, J=7.2 \mathrm{~Hz}, 3 \mathrm{H}), 1.27-1.36(\mathrm{~m}, 2 \mathrm{H}), 1.44-1.52(\mathrm{~m}$, 2H), 2.25-2.39 (m, 2H), $3.57(\mathrm{~d}, J=7.1 \mathrm{~Hz}, 2 \mathrm{H}), 4.62(\mathrm{t}, J=7.1 \mathrm{~Hz}, 1 \mathrm{H}), 7.41-7.62(\mathrm{~m}, 5 \mathrm{H})$, 7.88-7.91 (m, 2H), 8.14-8.17 (m, 2H); ${ }^{13} \mathrm{C}$ NMR $\delta$ 13.9, 22.3, 31.5, 31.7, 44.1, 45.4, 123.9 (2C), 128.2 (2C), 129.1 (2C), 129.2 (2C), 133.9, 136.7, 147.3, 150.7, 196.6. Anal. calcd for $\mathrm{C}_{19} \mathrm{H}_{21} \mathrm{O}_{3} \mathrm{SN}$ : C, 66.45; H, 6.16; N, 4.08. Found: C, 66.51; H, 6.03; N, 3.92.

3-(4-Chlorothiophenyl)-3-(4-nitrophenyl)-1-phenylpropan-1-one (entry 9). White crystal; m.p. 95-97 ${ }^{\circ} \mathrm{C}$ (hexane-ether 4:1); IR 3058, 1681, 1519, 1346, $688 \mathrm{~cm}^{-1}$; ${ }^{1} \mathrm{H}$ NMR $\delta 3.65(\mathrm{~d}, J=$ $7.1 \mathrm{~Hz}, 2 \mathrm{H}), 4.94(\mathrm{t}, J=7.1 \mathrm{~Hz}, 1 \mathrm{H}), 7.15-7.61(\mathrm{~m}, 9 \mathrm{H}), 7.88-7.91(\mathrm{~m}, 2 \mathrm{H}), 8.09-8.12(\mathrm{~m}, 2 \mathrm{H})$; ${ }^{13} \mathrm{C}$ NMR $\delta 44.3,48.5,124.2$ (2C), 128.2 (2C), 128.4, 128.8 (2C), 128.9 (2C), 129.4 (2C), 131.6, 132.2, 134.1, 135.1 (2C), 136.6, 149.3, 196.2. Anal. calcd for $\mathrm{C}_{21} \mathrm{H}_{16} \mathrm{O}_{3} \mathrm{SN}: \mathrm{C}, 69.60 ; \mathrm{H}, 4.45$; N, 3.86. Found: C, 69.80; H, 4.26; N, 4.09.

3-(4-Methoxyphenyl)-1-phenyl-3-thiobutylpropan-1-one (entry 10). Colorless oil; IR 3057, 2835, 1683, $1608 \mathrm{~cm}^{-1}$; ${ }^{1} \mathrm{H}$ NMR $\delta 0.84(\mathrm{t}, J=7.2 \mathrm{~Hz}, 3 \mathrm{H}), 1.26-1.35(\mathrm{~m}, 2 \mathrm{H}), 1.43-1.54(\mathrm{~m}$, 2H), 2.24-2.38 (m, 2H), 3.50 (d, J=7.1 Hz, 2H), $3.77(\mathrm{~s}, 3 \mathrm{H}), 4.52$ (t, $J=7.1 \mathrm{~Hz}, 1 \mathrm{H}), 6.84(\mathrm{~d}, J$ $=7.4 \mathrm{~Hz}, 2 \mathrm{H}), 7.26-7.56(\mathrm{~m}, 5 \mathrm{H}), 7.90(\mathrm{~d}, J=7.4 \mathrm{~Hz}, 2 \mathrm{H}) ;{ }^{13} \mathrm{C} \mathrm{NMR} \delta 14.1,22.4,31.5,31.7$, 44.1, 45.9, 55.6, 114.2, 114.3, 128.5 (2C), 129.0 (2C), 129.2, 129.3, 133.6, 134.6, 137.2, 158.9, 197.6. Anal. calcd for $\mathrm{C}_{20} \mathrm{H}_{24} \mathrm{O}_{2} \mathrm{~S}:$ C, 73.13; H, 7.36. Found: C, 73.01; H, 7.21.

1-Phenyl-3-(thiobutyl)butan-1-one (entry 13). Colorless oil; IR 3032, 2929, 1685, $1596 \mathrm{~cm}^{-1}$; ${ }^{1} \mathrm{H}$ NMR $\delta 0.91(\mathrm{t}, J=7.4 \mathrm{~Hz}, 3 \mathrm{H}), 1.35(\mathrm{~d}, J=6.7 \mathrm{~Hz}, 3 \mathrm{H}), 1.36-1.46(\mathrm{~m}, 2 \mathrm{H}), 1.53-1.64(\mathrm{~m}$, 2H), $2.57(\mathrm{t}, J=7.3 \mathrm{~Hz}, 2 \mathrm{H}), 3.05-3.33(\mathrm{~m}, 2 \mathrm{H}), 3.42-3.52(\mathrm{~m}, 1 \mathrm{H}), 7.26-7.59(\mathrm{~m}, 3 \mathrm{H}), 7.90-7.97$ 
(m, 2H); ${ }^{13} \mathrm{C}$ NMR $\delta 14.1,22.2,22.5,31.0,32.2,35.9,46.6,128.5$ (2C), 129.1 (2C), 133.3, 137.4, 198.7. Anal. calcd for $\mathrm{C}_{14} \mathrm{H}_{20} \mathrm{OS}: \mathrm{C}, 71.14 ; \mathrm{H}, 8.53$. Found: C, 70.96; H, 8.45.

1-Phenyl-3-(2-aminothiophenyl)butan-1-one (entry 15). Colorless oil; IR 3460, 3361, 2927, 1683, 1452, $692 \mathrm{~cm}^{-1}$; ${ }^{1} \mathrm{H}$ NMR $\delta 1.36(\mathrm{~d}, J=6.7 \mathrm{~Hz}, 3 \mathrm{H}), 2.55\left(\mathrm{dd}, J_{1}=10.6 \mathrm{~Hz}, J_{2}=12.8 \mathrm{~Hz}\right.$, $1 \mathrm{H}), 3.06\left(\mathrm{dd}, J_{1}=5.3, J_{2}=12.8 \mathrm{~Hz}, 1 \mathrm{H}\right), 4.00-4.12(\mathrm{~m}, 1 \mathrm{H}), 7.03-7.59(\mathrm{~m}, 7 \mathrm{H}), 8.04-8.07(\mathrm{~m}$, $2 \mathrm{H}) ;{ }^{13} \mathrm{C}$ NMR $\delta 22.3,38.1,53.0,124.9,125.3,127.8$ (2C), 128.5 (2C), 129.1, 131.4, 135.7, 138.7, 153.1, 170.7, 198.7. Anal. calcd for $\mathrm{C}_{16} \mathrm{H}_{17} \mathrm{NOS}: \mathrm{C}, 70.82$; H, 6.31; N, 5.16. Found: C, 70.69; H, 6.23; N, 5.01.

\section{Acknowledgements}

This investigation has enjoyed financial support from CSIR [grant No. 01(1739)/02], New Delhi. S.S.D. and S.S. are also thankful to CSIR for their fellowships.

\section{References}

1. (a) Zahouily, M.; Abrouki, Y.; Rayadh, A. Tetrahedron Lett. 2002, 43, 7729. (b) Abrouki, Y.; Zahouily, M.; Rayadh, A.; Bahlaouan, B.; Sebti, S. Tetrahedron Lett. 2002, 43, 8951. (c) Bandini, M.; Cozzi, P. G.; Giacomini, M.; Melchiorre, P.; Selva, S.; Umani-Ronchi, A. J. Org. Chem. 2002, 67, 3700. (d) Zahouily, M.; Abrouki, Y.; Rayadh, A.; Sebti, S.; Dhimane, H.; David, M. Tetrahedron Lett. 2003, 44, 2463.

2. (a) Ghosh, R. Indian J. Chem. 2001, 40B, 550. (b) Chauhan, K. K.; Frost, C. G. J. Chem. Soc., Perkin Trans. 1 2000, 3015. (c) Loh, T. P.; Liung, S. B. K. -W.; Tan, K. -L.; Wei, L. L. Tetrahedron 2000, 56, 3227.

3. (a) Ranu, B. C. Eur. J. Org. Chem. 2000, 2347. (b) Ranu, B. C.; Hajra, A.; Jana, U. J. Org. Chem. 2000, 65, 6270. (c) Ranu, B. C.; Hajra, A.; Jana, U. Tetrahedron Lett. 2000, 41, 531. (d) Ranu, B. C.; Samanta, S.; Hajra, A. Synlett 2002, 987. (e) Ranu, B. C.; Das, A.; Samanta, S. Synlett 2002, 727. (f) Ranu, B. C.; Dey, S. S.; Hajra, A. Tetrahedron 2002, 58, 2529. (g) Ranu, B. C.; Hajra, A.; Dey, S. S.; Jana, U. Tetrahedron 2003, 59, 813.

4. (a) Loh, T. -P.; Wei, L. -L. Tetrahedron 1998, 54, 7615. (b) Loh, T. -P.; Wei, L. -L. Synlett 1998, 975. (c) Yadav, J. S.; Abraham, S.; Reddy, B. V. S.; Sabitha, G. Tetrahedron Lett. 2001, 42, 8063. (d) Yadav, J. S.; Abraham, S.; Reddy, B. V. S.; Sabitha, G. Synthesis 2001, 2165.

5. Ranu, B. C.; Majee, A. Chem. Commun. 1997, 1225.

6. Katritzky, A. R.; Chen, J.; Balyakov, S. A. Tetrahedron Lett. 1996, 37, 6631.

7. Alam, M. M.; Varala, R. V.; Adapa, S. R. Tetrahedron Lett. 2003, 44, 5115.

8. Ahuja, P. R.; Natu, A. A.; Gogte, V. N. Tetrahedron Lett. 1980, 21, 4743. 
9. Furniss, B. S.; Hannaford, A. J.; Smith, P. W. G.; Tatchell, A. R. Vogel's Text Book of Practical Chemistry, $5^{\text {th }}$ Edn.; Longman: England, 1989, p 1034. 